\title{
Effect of biorational and selective insecticides on transmission of Candidatus Liberibacter solanacearum to potato plants
}

\author{
A.M. Barnes, J. Vereijssen, S.E. Thompson and R.C. Butler \\ The New Zealand Institute for Plant E Food Research Limited, Private Bag 4704, \\ Christchurch 8140, New Zealand \\ Corresponding author: anna-marie.barnes@plantandfood.co.nz
}

\begin{abstract}
The tomato potato psyllid, Bactericera cockerelli (Šulc) (TPP), vectors Candidatus Liberibacter solanacearum (CLso), which is the putative causative agent of zebra chip, an important disease of potato. Laboratory bioassays using potted potato plants and CLsopositive TPP were conducted to assess the potential of biorational and selective insecticides to disrupt CLso transmission. The first assay tested eight products, with TPP introduced to plants $24 \mathrm{~h}$ after insecticide application and sprayed off after $24 \mathrm{~h}$. The second assay further tested five products and assessed systemic activity, with TPP introduced 24, 72 or $168 \mathrm{~h}$ after product application. Stolon samples were collected and analysed for presence of the CLso gene using qPCR. Results have shown potential for biorational and selective insecticides to reduce the quantity of CLso transmitted to plants with varying efficiencies, and although none completely inhibited CLso transmission, there is scope for these products to be included in crop management strategies.
\end{abstract}

Keywords tomato potato psyllid, CLso transmission, vector management, biorational and selective insecticides.

\section{INTRODUCTION}

Following the incursion of the tomato potato psyllid, Bactericera cockerelli (Šulc) (TPP), a serious pest of solanaceous plants, into New Zealand in 2006, there has been a significant increase in insecticide applications in New Zealand's economically important solanaceous crops. This increase jeopardises the successful implementation of Integrated Pest Management (IPM) programmes (Teulon et al. 2009). TPP not only causes mechanical feeding damage, but also vectors the bacterial pathogen Candidatus Liberibacter solanacearum (CLso), the putative cause of 'zebra chip' disease in potato tubers
(Munyaneza et al. 2007; Pitman et al. 2011).

Management of insect vectors often requires a multi-pronged approach in which several pathways (vector - plant host; pathogen - plant host) are interrupted to keep the plant disease acceptably low (Jones et al. 2010). Previous research has shown that some selective and biorational insecticides have potential to disrupt TPP feeding and behaviour, and increase mortality (Dohmen-Vereijssen et al. 2012; Gardner-Gee et al. 2012; Jorgensen et al. 2013).

This study looked at the vector-plant host pathway with the aim of investigating the 
potential of selected biorational insecticides and some selective insecticides to disrupt transmission of CLso by TPP.

\section{MATERIALS AND METHODS}

Two controlled-environment growth room trials were conducted. The main trial in MarchApril 2013 tested eight treatments, with TPP introduced to plants $24 \mathrm{~h}$ after spray application. Five of the treatments were evaluated further in July-August and September-October 2013 in an additional trial with TPP introduced $24 \mathrm{~h}, 72 \mathrm{~h}$ and $168 \mathrm{~h}$ post spraying to test any systemic activity of the products (Table 1).

\section{Insect source and rearing}

Adult TPP used in the trials were sourced from a laboratory colony at Plant \& Food Research, Lincoln, Canterbury. TPP were reared on tomato plants (Lycopersicon esculentum 'Money Maker') in a controlled environment growth room at $25^{\circ} \mathrm{C}$, 16:8 h light:dark and 41\% RH. The CLso status of this colony was determined by destructive quantitative polymerase chain reaction (qPCR) testing of individual insects before psyllids from the colony were used in the trials and confirmed to be CLso positive (data not shown). The insects were not sexed before use in the trials.

\section{Plant material}

In vitro tissue culture potato plantlets (Solanum tuberosum 'Russet Burbank' - guaranteed CLso free by Aspara Pacific Ltd, Lincoln, Canterbury) were transplanted into individual 1.5-litre black plastic pots containing standard potting mix and placed in white 2-litre plastic containers to allow watering. Plants were then grown to vegetative growth stage (10-16 leaves) over 5 weeks in a controlled environment growth room (see above). Plants were watered twice weekly. Immediately before insecticide application, 20 plants were selected at random and leaf material collected for qPCR testing, which confirmed the plants' CLso-negative status.

\section{Insecticide selection and application}

After the 5-week growth period, whole individual potato plants were sprayed with either a biorational or selective insecticide, water (negative control) or Avid ${ }^{\circledR}$ (positive control).
Insecticide selection was based on previous results obtained in the research programme (Berry \& Bourhill 2012; Dohmen-Vereijssen et al. 2012; Gardner-Gee et al. 2012; Barnes et al. 2013). Two litres of each product in each of the trials (Table 1) was prepared according to label rates and applied to the plants to the point of runoff via a 2 -litre pressurised hand sprayer.

\section{Introduction of tomato potato psyllids to plants}

Twenty-four hours after products were applied to the potato plants, individual plants were enclosed within mesh bags. In the main trial, ten CLso-positive insects were introduced to each individually bagged potato plant $24 \mathrm{~h}$ after spray application. In the additional trial, ten CLsopositive insects were introduced to each potato plant $24 \mathrm{~h}, 72 \mathrm{~h}$ or $168 \mathrm{~h}$ after spray application. The additional trial was run in eight replicates, achieved in two separate runs (each of four replicates) at different time periods, because of growth room space and availability. The bags were secured around the base of each pot with tape. TPP were subsequently left on the plants for a 24 -h period, after which the adults and eggs were sprayed with Avid, allowing the plants to continue to grow without psyllids or continued CLso pressure from the psyllids.

Stolon samples were taken from the potato plants 4 weeks after the TPP were sprayed off. Approximately 1-2 cm of stem tissue was removed from the portion of the main stem found just below soil level, using a pre-sterilised scalpel, which was re-sterilised between plants. If tubers were also present, approximately $3 \mathrm{~cm}$ of stolon tissue was excised. Each of the tissue samples was immediately frozen at $-20^{\circ} \mathrm{C}$ prior to $\mathrm{qPCR}$ analysis.

To prepare the stem and stolon samples for qPCR analysis, the soiled outer skin was scraped off. Approximately $100 \mathrm{mg}$ of material was obtained for DNA extraction and purification as described by Beard et al. (2013). Quantities of the CLso gene (using primers LsoF/Lso16SF/ Lso16SR1, designed against the gene encoding $16 \mathrm{~S}$ ribosomal RNA) and the reference or 'housekeeping' gene Ef1 $\alpha$ (primer pair EF1aF/ EF1aR), which is naturally present in the plant and is expected to be present in the same quantity 


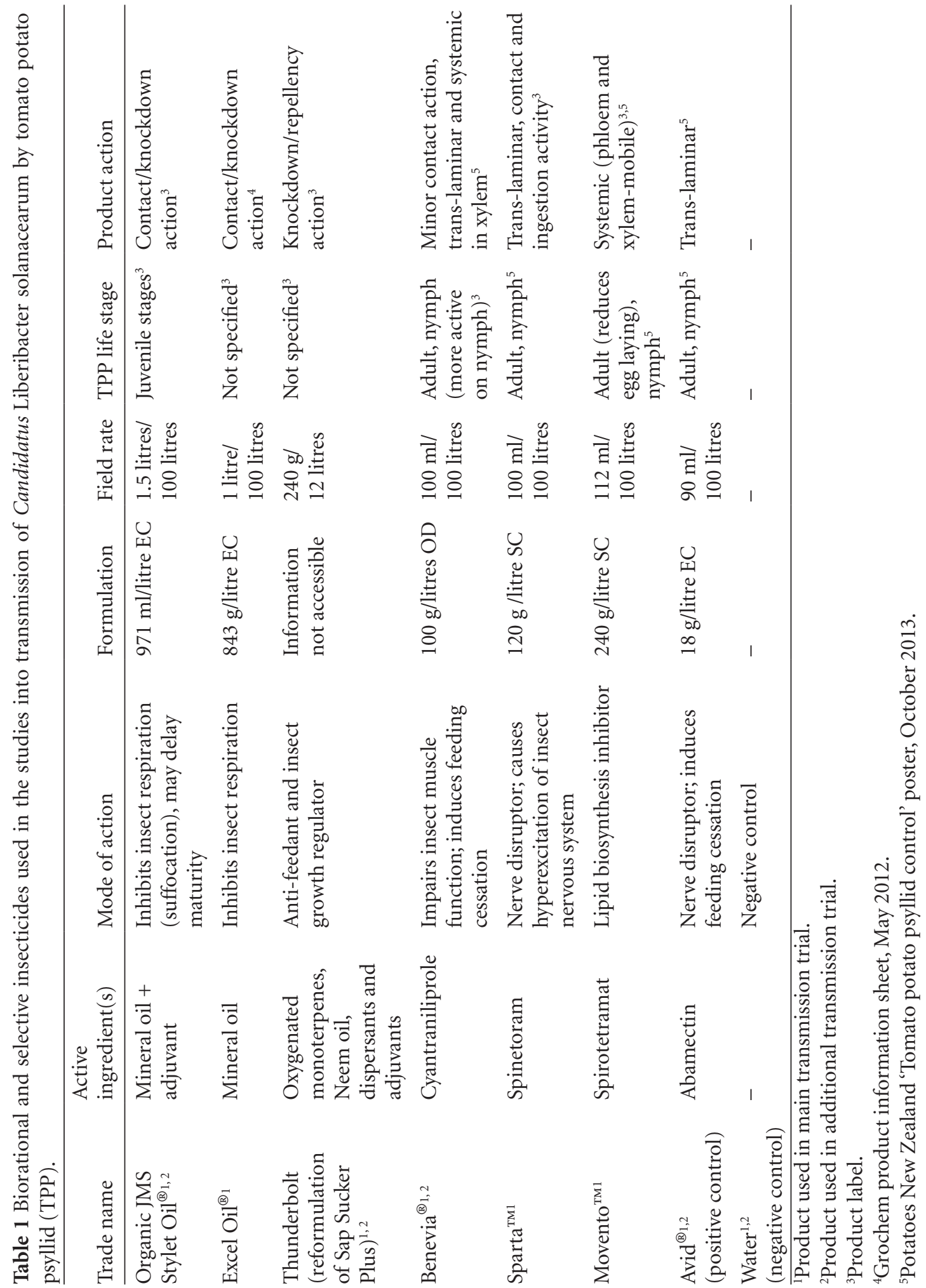


in each cell, were estimated by qPCR as described by Beard et al. (2013).

\section{Trial design and statistical analysis}

In the main trial, each treatment was replicated four times (32 pots in total) and laid out in a Latinized resolvable block design. In the additional trial, each treatment was replicated eight times (120 pots total), laid out in a resolvable row-column design (CycSoftware 2009). Controlled environment growth room conditions were as described above.

qPCR was used to estimate the quantity of the CLso gene and the reference gene Efl $\alpha$. Calibration regression equations were used to convert cycle threshold $(\mathrm{Ct})$ numbers to quantities. Wells with 'undetermined' $\mathrm{Ct}$ were assumed to have quantity $=0$. The ratio of CLso to $\mathrm{EF} 1 \alpha$ quantities is the primary measurement of interest, since this provides CLso quantities corrected for any variation in the amount of plant material processed (Beard et al. 2013).

A Poisson-Gamma hierarchical generalised linear model approach (HGLM) (Lee et al. 2006) was used to analyse the quantities, with data for the two genes (CLso and EF1 $\alpha$ ) analysed simultaneously. This allowed the ratio between the two genes to be obtained directly from an analysis of the quantities, in addition to the means for the individual genes. In the analysis, random effects (such as spatial factors) were included with a gamma distribution and a log link, and the fixed effects (treatments, insect application time, gene and the interactions between these) with a Poisson distribution and log link. The importance of the random effects was assessed with a $\chi^{2}$ test of the change in likelihood on dropping the effect, as implemented in the HGRTEST procedure, and fixed effects with a similar test using the HGFTEST procedure. In the results presented, means were obtained on the transformed (link) scale along with 95\% confidence limits and were back-transformed for presentation. All analyses were carried out with GenStat (release 16).

\section{RESULTS}

\section{Main trial}

There was no treatment for which all CLso values were undetermined or missing, indicating that the CLso quantity was not completely zero for any treatment. DNA quantities did not vary substantially between the treatments for the reference gene $\mathrm{EF} 1 \alpha(\mathrm{P}=0.898)$, but there were some significant treatment differences in quantities for the CLso gene $(\mathrm{P}=0.028)$ and thus also for the ratio between the two genes $(\mathrm{P}=0.051)$.

CLso quantities and ratios were significantly lower than those with water for Excel Oil and Avid treatments $(\mathrm{P}<0.05)$, and only water and Benevia treatment had significantly higher quantities than found with Avid treatment $(\mathrm{P}<0.05)$ (Figure 1).

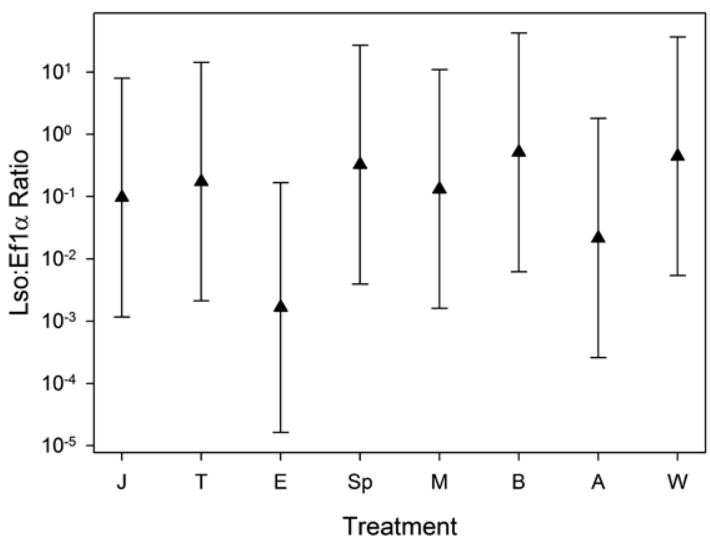

Figure 1 The mean ratio between Candidatus Liberibacter solanacearum (CLso) and EF1 $\alpha$ genes for each treatment in the main trial, where a smaller ratio indicates a lower quantity of CLso gene present in the potato sample. Error bars are approximate $95 \%$ confidence intervals for the means. $\mathrm{J}=$ Organic JMS Stylet-Oil ${ }^{\circledR}, \mathrm{T}=$ Thunderbolt, $\mathrm{E}=$ Excel Oil $^{\circledR}, \mathrm{Sp}=$ Sparta $^{\mathrm{TM}}, \mathrm{M}=$ Movento $^{\mathrm{TM}}, \mathrm{B}=$ Benevia $^{\circledR}, \mathrm{A}=$ Avid $^{\circledR}, \mathrm{W}=$ Water.

\section{Additional trial}

There was no treatment for which all values were undetermined, indicating that the CLso quantity was not completely zero for any treatment. There were no significant treatment or time differences in the quantities for the two genes or the ratio between them (Figure 2).

\section{DISCUSSION}

The main transmission trial showed that biorational and selective insecticides can decrease transmission of CLso to potato plants by TPP, 


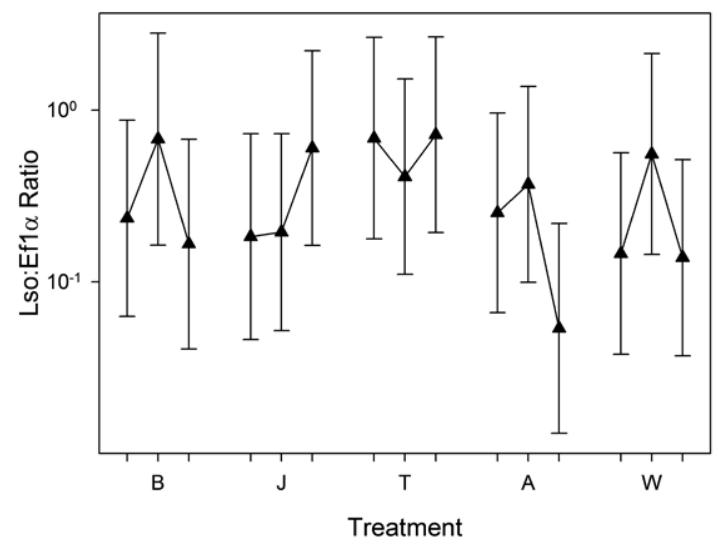

Figure 2 The mean ratio between Candidatus Liberibacter solanacearum (CLso) and EF1 $\alpha$ genes for each treatment at each of the three tomato potato psyllid (TPP), application times in the additional trial (ticks on $\mathrm{X}$-axis indicate 1,3 , and 7 days after treatment). A smaller ratio indicates a lower quantity of CLso gene present in the sample. Error bars are approximate 95\% confidence intervals for the means. $\mathrm{B}=$ Benevia $^{\circledR}$, $\mathrm{J}=$ Organic JMS Stylet-Oil ${ }^{\circledR}, \mathrm{T}=$ Thunderbolt, $^{\circ}$ $\mathrm{A}=$ Avid $^{\circledR}, \mathrm{W}=$ Water.

with the most effective treatments being Excel Oil (biorational) and Avid (selective), although no treatment tested completely prevented transmission. Excel Oil had performed well in previous bioassays (Dohmen-Vereijssen et al. 2012; Gardner-Gee et al. 2012) and the reduced transmission observed in the main trial is a promising result. Avid is a non-systemic, translaminar product and can take up to 7 days to reach maximum effectiveness. Its mode of action consists of paralysing the insect via nerve disruption and also causing feeding disruption, which may have led to the results observed. In the additional trial, there was no product that performed well overall and it is difficult to link these results to the mode of action of the products.

The results observed with Benevia may be due to the fact that Benevia, although being systemic, is xylem mobile. TPP is a phloem feeder, but does access the xylem (Pearson et al. 2010). However, this is unpredictable and only a small percentage of insects feed on xylem, although this may be more common when insects are transferred from one host plant species to another (M. Sandanayaka,
Plant \& Food Research, personal communication). As the adult TPP used were initially reared on tomato and then moved to potato for the duration of the trials, some xylem feeding could have been expected. The largest reduction in transmission was at 7 DAT (Figure 2), which can be explained by the systemic mode of action, requiring time to translocate in the plant. The product label states that rapid feeding cessation results within a few hours of exposure, but the time to death may be 3-6 days. Cyantraniliprole (active ingredient of Benevia) has been shown to increase mortality in Asian citrus psyllid (Diaphorina citri) (Tiwari \& Stelinski 2013) and TPP as well as induce reduced egg-laying in TPP (Gardner-Gee et al. 2012). It is believed that inhibition of CLso transmission has not previously been tested.

The quantity of CLso actually transmitted to a plant depends on the feeding behaviour of individual TPP, the number of TPP feeding on a plant and the CLso titre, which varies between individual insects. The two trials were carried out at different times of the year, and the amount of CLso present in the parent colony can fluctuate over time. This could explain the absence of significant effects in the additional trial, which was carried out at a different time of year to the main trial, with each of the runs for the additional trial also carried out 10 weeks apart. Also, significant differences found in laboratory trials do not always translate into significant effects in the field - in this situation not every TPP carries CLso and therefore not all can transmit the bacterium to plants and not all plants or tubers need to be free of CLso for a crop to be accepted for processing at the factory.

No biorational or selective insecticide assessed completely inhibited CLso transmission to potato plants. However, there is scope for these products to be included in crop management programmes. Other management strategies that interrupt vector-pathogen-host plant interactions include cultural practices (adjusted planting dates to escape highest TPP pressure, volunteer management, use of certified seed stock) and the initiation of spray programmes triggered by crop monitoring with sticky traps and/or manual plant assessments. A targeted approach to crop management utilising a combination of these strategies may lead to better management of TPP and a reduction in CLso-infected plants/tubers. 


\section{ACKNOWLEDGEMENTS}

This work was funded through the Ministry for Primary Industries Sustainable Farming Fund SFF11/058 IPM Tools for Psyllid Management programme. The authors would like to thank Melanie Walker and Stacey Skill (PFR Lincoln) and visiting scientist Phyllis Weintraub (Agricultural Research Organization, Israel) for technical assistance, Melanie Davidson and Grant Smith (PFR Lincoln) for helpful comments on the manuscript and Robin Gardner-Gee and Manoharie Sandanayaka (PFR Mt Albert) for useful discussions and sharing of information.

\section{REFERENCES}

Barnes AM, Butler RC, Vereijssen J 2013. Effect of selected biorational insecticides and conventional insecticides on transmission of Candidatus Liberibacter solanacearum by tomato potato psyllid (Bactericera cockerelli) on potato plants. Report to Potatoes New Zealand, Plant \& Food Report No. 8516, Plant \& Food Research, Lincoln, New Zealand. 23 p.

Beard SS, Pitman AR, Kraberger S, Scott IAW 2013. SYBR Green real-time quantitative PCR for the specific detection and quantification of 'Candidatus Liberibacter solanacearum' in field samples from New Zealand. European Journal of Plant Pathology 136: 203-215.

Berry NA, Bourhill A 2012. Review of soft chemical options and research for insect pest control. Report to Potatoes New Zealand, Plant \& Food Report No. 6065, Plant \& Food Research, Lincoln, New Zealand. 24 p.

CycSoftware 2009. CycDesigN 4.0 A package for the computer generation of experimental designs. Version 4.0, CycSoftware Ltd, Hamilton, New Zealand.

Dohmen-Vereijssen J, Jorgensen N, Butler RC 2012. SFF11/058: IPM tools for psyllid management - Soft chemical options. Report to The Sustainable Farming Fund, Plant \& Food Report No. 7377, Plant \& Food Research, Lincoln, New Zealand. 27 p.

Gardner-Gee R, Butler RC, Griffin M, Puketapu A, MacDonald F, Jamieson LE 2012. Effect of insecticide residues on the behaviour, mortality and fecundity of the tomato potato psyllid (Bactericera cockerelli: TPP). Plant \& Food Research, Auckland, New Zealand. http://www.tomatoesnz.co.nz/documents/ reports/98/7392\%20-\%20Robin\%20GardnerGee\%20-\%20Effect\%20of\%20insecticide $\% 20$ residues $\% 20 \% 202013 \% 20$ PFR\%2048905\%20 FINAL\%20with\%20changes\%2010DEC12. pdf (accessed: 25 March 2014).

Jones RAC, Salam MU, Maling TJ, Diggle AJ, Thackray DJ 2010. Principles of predicting plant virus disease epidemics. Annual Review of Phytopathology 48: 179-203.

Jorgensen N, Butler RC, Vereijssen J 2013. Biorational insecticides for control of the tomato potato psyllid. New Zealand Plant Protection 66: 333-340.

Lee Y, Nelder JA, Pawitan Y 2006. Generalized linear models with random effects: Unified analysis via h-likelihoods. Monographs on Statistics and Applied Probability. Chapman \& Hall/CRC Press, London, UK. 416 p.

Munyaneza JE, Crosslin JM, Upton JE 2007. Association of Bactericera cockerelli (Homoptera : Psyllidae) with "zebra chip," a new potato disease in southwestern United States and Mexico. Journal of Economic Entomology 100: 656-663.

Pearson CC, Backus EA, Munyaneza JE 2010. Feeding biology of the potato psyllid Bactericera cockerelli. The 10th Annual Zebra Chip Reporting Session, 7-10 November 2010, Dallas, Texas, USA. Pp. 178-182.

Pitman AR, Drayton GM, Kraberger SJ, Genet RA, Scott IAW 2011. Tuber transmission of 'Candidatus Liberibacter solanacearum' and its association with zebra chip on potato in New Zealand. European Journal of Plant Pathology 129: 389-398.

Teulon DAJ, Workman PJ, Nielsen M-C, Thomas KL, Anderson DP 2009. Bactericera cockerelli: incursion, dispersal and current distribution on vegetable crops in New Zealand. New Zealand Plant Protection 62: 136-144.

Tiwari S, Stelinski LL 2013. Effects of cyantraniliprole, a novel anthranilic diamide insecticide, against Asian citrus psyllid under laboratory and field conditions. Pest Management Science 69: 1066-1072. 